\title{
Insatisfação com a imagem corporal e relação com o nível de atividade física e estado nutricional em universitários
}

\author{
Body image dissatisfaction and its relationship with physical activity and \\ nutritional status in university students
}

\author{
E.P. Ferrari, A.P. Gordia, C.R. Martins, D.A. Silva, T.M. Quadros, E.L. Petroski
}

ARTIGO ORIGINAL | ORIGINAL ARTICLE

\begin{abstract}
RESUMO
O objetivo deste estudo foi verificar a associação da insatisfação com a imagem corporal, com o nível de atividade física e o estado nutricional em universitários recém-ingressos em uma universidade pública brasileira. Participaram do estudo 832 universitários (485 do sexo masculino) com média de 20.1 (desvio padrão $=4.6$ ) anos de idade. A massa corporal e a estatura autorrelatadas foram utilizadas para o cálculo do índice de massa corporal. Os universitários responderam o Body Shape Questionnaire e o International Physical Activity Questionnaire. Utilizou-se o teste exato de Fisher, considerando $p<.05$. As prevalências de insatisfação com a imagem corporal e inatividade física foram $10.1 \%$ e $14.5 \%$, respectivamente. Não houve associação significante entre a imagem corporal e o nível de atividade física. A insatisfação com a imagem corporal esteve associada ao estado nutricional para ambos os sexos $(p<$ .05). Conclui-se que os universitários com excesso de peso devem ser motivados a adquirirem um estilo de vida mais saudável, promovendo adequações no seu estado nutricional e também melhorando a sua imagem corporal.

Palavras-chave: atividade física, imagem corporal, estado nutricional, jovens universitários
\end{abstract}

ABSTRACT

The aim of the present study was to evaluate the association of body image dissatisfaction with physical activity level and nutritional status in freshmen from a public Brazilian university. A total of 832 university students $(485$ men) with a mean age of 20.1 years (standard deviation $=4.6$ ) participated in the study. Self-reported body weight and height were used for the calculation of body mass index. The students responded to the Body Shape Questionnaire and International Physical Activity Questionnaire. Data were analyzed using Fisher's exact test, considering $p<.05$. The prevalence of body image dissatisfaction and physical inactivity was $10.1 \%$ and $14.5 \%$, respectively. No significant association was observed between body image dissatisfaction and physical activity level. Body image dissatisfaction was associated with nutritional status in both genders $(p<.05)$. University students with excess body weight should be encouraged to pursue a healthier lifestyle in order to promote an adequate nutritional status and also to improve their body image.

Keywords: physical activity, body image, nutritional status, college students

Submetido: 03.05.2011 | Aceite: 29.08.2012

Elisa Pinheiro Ferrari, Alex Pinheiro Gordia, Cilene Rebolho Martins, Diego Augusto Santos Silva, Teresa Maria Bianchini de Quadros e Edio Luiz Petroski. Departamento de Educação Física, Centro de Desportos, Universidade Federal de Santa Catarina, Florianópolis, SC, Brasil.

Endereço para correspondência: Elisa Pinheiro Ferrari, Universidade Federal de Santa Catarina, Campus Trindade, Florianópolis, SC, Brasil.

E-mail: elisaferrari_@hotmail.com 
Ao longo das últimas décadas, o crescente processo de modernização conduziu a população a um estilo de vida inadequado, caracterizado pelo aumento do consumo de alimentos com alto teor calórico e declínio no nível de atividade física (NAF) (Frutuoso, Bismarck-Nasr, \& Gambardella, 2003). Este padrão de comportamento resulta em um aumento nas prevalências de sobrepeso e obesidade em todos os estratos da população (Ribeiro et al., 2006).

O estilo de vida inadequado não implica somente em efeitos negativos sobre a saúde física, mas também sobre a saúde mental, estimulando a ocorrência de sintomas de estresse, ansiedade e depressão (Ferreira, Tufik, \& Mello, 2001), podendo interferir, também na percepção corporal do indivíduo, tornando-o insatisfeito com a imagem corporal (IC).

Estudos prévios relatam que a prática da atividade física está associada à melhoras na IC (Vieira, 2004; Williams \& Cash, 2001). Vieira (2004), ao analisar o efeito da atividade física em academias na imagem corporal de indivíduos obesos, identificou que após a intervenção, a prevalência de insatisfação com a IC foi menor em comparação ao pré-teste. O mesmo foi observado por Williams e Cash (2001), ao avaliar a eficiência de um programa de treinamento com pesos em estudantes universitários. Contrariamente, em um estudo com homens e mulheres fisicamente ativos, não foi encontrada associação entre atividade física e IC (Davis \& Cowles, 1991). O autor comenta que esse resultado se deve à obsessão pelas formas corporais magras, o que leva os indivíduos a permanecerem insatisfeitos com a IC. Assim, além da escassez de estudos sobre a relação entre NAF e insatisfação com a IC, sobretudo, em universitários, percebe-se uma falta de consenso na literatura a respeito dessa questão.

Outro fator que pode influenciar a IC é o estado nutricional, devido à imposição cultural de corpos extremamente magros para o sexo feminino (Schwartz \& Brownell, 2004) e musculosos para o masculino (Damasceno, Lima, Vianna, Vianna, \& Novaes, 2005), como forma subliminar de sucesso, felicidade, dinamismo e bem-estar pessoal (Andrade \& Bosi, 2003). Diante disso, aqueles indivíduos que não conseguem atingir este padrão corporal estão propensos a desenvolver uma percepção negativa da IC (Stipp \& Marques de Oliveira, 2003).

Em uma pesquisa realizada com universitários (Kakeshita \& Almeida, 2006), os autores verificaram que aqueles com excesso de peso apresentaram maior insatisfação com a IC. Entretanto, outro estudo realizado com essa população evidenciou que mesmo os estudantes com o peso corporal adequado manifestavam um nível elevado de insatisfação com a IC (Bosi, Uchimura, \& Luiz, 2008). Desta forma, evidencia-se a necessidade de verificar a relação entre insatisfação com a IC e estado nutricional, para que este conhecimento possa apontar a direção a ser seguida no sentido de promover melhoras na IC e na aceitação do próprio corpo em indivíduos que estão em processo de formação universitária.

O final da adolescência e o início da fase adulta caracterizam períodos em que as modificações no estado nutricional, o NAF e a percepção da IC são muito evidenciadas (França \& Colares, 2008), assim como as relações entre estes três fatores. Dentre os aspectos determinantes destas mudanças, destaca-se o ingresso na universidade, período no qual o indivíduo adquire novas relações sociais, que, somado às obrigações acadêmicas, pode levar a condutas de saúde menos saudáveis. Assim, torna-se relevante a investigação dessas variáveis e suas associações neste segmento da população, a fim de orientar o desenvolvimento de programas de promoção da saúde, visando a aquisição e manutenção de comportamentos saudáveis.

Desta forma, o objetivo do presente estudo foi verificar a associação da insatisfação com a IC com o NAF e o estado nutricional em univer- 
sitários recém-ingressos em uma universidade pública brasileira.

\section{MÉTODO}

\section{Amostra}

Este estudo foi realizado com base no banco de dados do projeto de pesquisa "Avaliação da aptidão física relacionada à saúde de universitários da UFSC". O protocolo do estudo foi aprovado pelo Comitê de Ética em Pesquisa da Universidade Federal de Santa Catarina (Processo n 096/2007).

A população de estudo foi composta por 2290 universitários ingressantes no primeiro semestre do ano letivo de 2008 na Universidade Federal de Santa Catarina. Para o cálculo do tamanho da amostra, foi utilizada a metodologia recomendada por Thomas, Nelson, e Silverman (2007), considerando um nível de confiança igual a 95\% e um erro máximo permitido de três pontos percentuais. A amostragem estratificada proporcional foi utilizada por centro de ensino e por turno de estudo (diurno e noturno). Foi utilizado um procedimento randomizado para o sorteio das turmas dentro de cada centro de ensino. Com base no cálculo amostral, estimou-se que seria necessário avaliar 728 universitários, no entanto, devido à coleta de dados ser realizada por conglomerado de turmas, sendo convidados a participarem do estudo todos os estudantes presentes em sala de aula, no dia da coleta, desta forma, responderam o questionário 921 indivíduos.

Foram excluídos do estudo 89 indivíduos por não informarem dados referentes às variáveis analisadas (sexo, NAF, estatura, massa corporal e IC). Desta forma, a amostra final foi composta por 832 universitários (485 do sexo masculino e 347 do sexo feminino), com média de idade de 20.1 (desvio padrão $=4.6$ ) anos.

\section{Instrumentos e Procedimentos}

A massa corporal e a estatura foram obtidas através de medidas autorreferidas. A validade destas medidas tem sido comprovada com a população adulta brasileira (Coqueiro, Borges, Araújo, Pelegrini, \& Rodrigues, 2009). Para classificação do IMC utilizaram-se os seguintes pontos de corte: baixo peso $(\leq 18.5)$, peso normal ( $>18.5$ e $\leq 24.9)$ e excesso de peso $(\geq$ 25) (sobrepeso + obesidade) estabelecido pela Organização Mundial da Saúde (World Health Organization [WHO], 1998). Os indivíduos com sobrepeso e obesidade foram agrupados formando a categoria excesso de peso.

A insatisfação com a IC foi avaliada por meio do Body Shape Questionnaire (BSQ - 34). Este instrumento foi traduzido e validado em universitários por Di Pietro e Silveira (2009), apresentando uma consistência interna de 0.97 e uma solução da escala fatorial de quatro dimensões, representando $66.4 \%$ da variabilidade total dos dados. O BSQ-34 é um questionário autoaplicável do tipo escala Likert, composto por 34 perguntas, com seis opções de resposta (nunca, raramente, às vezes, frequentemente, muito frequentemente e sempre) que pontuam de um a seis, sendo a maior pontuação conferida àquelas respostas que refletem maior preocupação com a IC e maior autodepreciação devido à aparência física, especialmente no sentido de sentir-se com excesso de peso. A partir da pontuação obtida, os indivíduos foram classificados em satisfeitos (escore $<111$ ) ou insatisfeitos (escore $\geq 111$ ) (Alves, Vasconcelos, Calvo, \& Neves, 2008).

O NAF foi mensurado através do Questionário Internacional de Atividade Física (International Physical Activity Questionnaire - IPAQ; versão 8 , forma curta, última semana). Para avaliar o NAF dos universitários, foi utilizada a classificação desenvolvida pelo Comitê de Pesquisas sobre o IPAQ (IPAQ Research Committee, 2005). O IPAQ foi validado para a população adulta brasileira por Matsudo et al. (2001). Este método leva em consideração os critérios de frequência e duração, dividindo os indivíduos em três categorias: inativo, moderadamente ativo e muito ativo. 


\section{Análise Estatística}

A estatística descritiva foi utilizada por meio da distribuição de frequências. Para verificar as associações entre as variáveis estudadas utilizou-se o teste exato de Fisher, com todas as análises controladas por sexo. Os dados foram analisados no programa estatístico SPSS 15.0, adotando-se um nível de significância de 5\%.

\section{RESULTADOS}

A prevalência de insatisfação com a IC foi de 10.1\%. Dos universitários insatisfeitos, $90.1 \%$ foram do sexo feminino e $9.9 \%$ do sexo masculino.

Quanto à inatividade física, a prevalência foi de $14.5 \%$, sendo que os homens apresentaram menor prevalência (11.9\%) em relação às mulheres (18.2\%).

$\mathrm{Na}$ Tabela 1 verifica-se que tanto no sexo masculino quanto no feminino não foi observada associação significativa entre IC e NAF ( $p$ $>$.05).

Quadro 1.

Associação entre a IC e o NAF em universitários de acordo com o sexo. Florianópolis, Santa Catarina, 2008.

\begin{tabular}{|c|c|c|c|}
\hline \multirow[b]{2}{*}{ NAF } & \multicolumn{2}{|c|}{ Imagem Corporal \% $(n)$} & \multirow[b]{2}{*}{$p$ valor } \\
\hline & Satisfeito & Insatisfeito & \\
\hline \multicolumn{4}{|c|}{ Sexo masculino } \\
\hline Inativo & $100.0(59)$ & $0.0(0)$ & \\
\hline $\begin{array}{l}\text { Moderadamente } \\
\text { ativo }\end{array}$ & $97.5(389)$ & $2.5(10)$ & 0.330 \\
\hline Muito ativo & $100.0(27)$ & $0.0(0)$ & \\
\hline \multicolumn{4}{|c|}{ Sexo feminino } \\
\hline Inativo & $85.5(53)$ & $14.5(9)$ & \\
\hline $\begin{array}{l}\text { Moderadamente } \\
\text { ativo }\end{array}$ & $77.4(216)$ & $22.6(63)$ & 0.280 \\
\hline Muito ativo & $66.7(4)$ & $33.3(2)$ & \\
\hline
\end{tabular}

Considerando a IC e o estado nutricional (Tabela 2), verificou-se uma associação significativa tanto para o sexo masculino quanto para o feminino $(p<.05)$. Observa-se que, em ambos os sexos, houve uma maior proporção de insatisfação com a IC nos indivíduos com excesso de peso.
Quadro 2.

Associação entre a IC e o estado nutricional em universitários de acordo com o sexo. Florianópolis, Santa Catarina, 2008.

\begin{tabular}{|c|c|c|c|}
\hline \multirow[b]{2}{*}{$\begin{array}{c}\text { Estado } \\
\text { nutricional }\end{array}$} & \multicolumn{2}{|c|}{ Imagem Corporal \% $(n)$} & \multirow[b]{2}{*}{$p$ valor } \\
\hline & Satisfeito & Insatisfeito & \\
\hline \multicolumn{4}{|c|}{ Sexo masculino } \\
\hline Baixo peso & $100.0(19)$ & $0.0(0)$ & \multirow{3}{*}{$0.001^{*}$} \\
\hline Peso normal & $98.9(368)$ & $1.1(4)$ & \\
\hline Excesso de peso & $93.6(88)$ & $6.4(6)$ & \\
\hline \multicolumn{4}{|c|}{ Sexo feminino } \\
\hline Baixo peso & $97.9(47)$ & $2.1(1)$ & \multirow{3}{*}{$0.001^{*}$} \\
\hline Peso normal & $78.5(208)$ & $21.5(57)$ & \\
\hline Excesso de peso & $52.9(18)$ & $47.1(16)$ & \\
\hline
\end{tabular}

$* p<.05$ (Teste Exato de Fisher).

\section{DISCUSSÃO}

De acordo com revisão prévia da literatura, nenhuma pesquisa foi encontrada, considerando a associação da IC com o NAF e o estado nutricional em universitários. Dessa forma, o presente estudo contribui para o corpo de conhecimento neste domínio, demonstrando que universitários com excesso de peso estão mais insatisfeitos com a IC, em relação àqueles com peso normal e baixo peso. Além disso, percebe-se que a insatisfação com a IC não está relacionada ao NAF nos universitários pesquisados.

Em relação à insatisfação com a IC, a prevalência encontrada no presente estudo foi inferior a apresentada por Luz (2003), o qual observou uma prevalência de insatisfação com a IC de $15.3 \%$ em universitários de Belo Horizonte, MG. A baixa prevalência encontrada na atual pesquisa pode estar relacionada à maior proporção de indivíduos do sexo masculino que compõem a amostra, uma vez que os homens tendem a apresentar maior insatisfação por magreza (Quadros et al., 2010) e o questionário utilizado, o BSQ, avalia somente a insatisfação por excesso de peso (Vieira, Sabadin, \& Marques de Oliveira, 2008). Diante disso, justifica-se, também, a menor prevalência de insatisfação com IC verificada no sexo masculino, 
em relação ao feminino, no presente estudo.

A partir dos resultados obtidos, destaca-se que a prevalência de inatividade física (14.5\%) está abaixo da identificada em outras investigações que utilizaram o IPAQ em universitários. Rodrigues, Cheik, e Mayer (2008) encontraram prevalência de inatividade física de $29.9 \%$ em universitários de Gurupi - TO. Entre acadêmicos de uma universidade pública de João Pessoa - PB, Fontes e Vianna (2009) identificaram uma prevalência de $31.2 \%$. Apesar da prevalência de inatividade física encontrada ser inferior a outras investigações, a elaboração de ações voltadas para a motivação à prática de atividade física torna-se necessária, a fim de que os universitários sejam estimulados a se inserirem ou permanecerem em programas de atividade física.

Ao comparar as prevalências de inatividade física entre os sexos, observou-se que a mesma foi superior no sexo feminino em relação ao masculino. Este resultado converge com o de Silva et al. (2007) que, ao avaliarem o NAF de estudantes universitários de Juiz de Fora MG, verificaram que $32 \%$ das mulheres eram inativas, enquanto somente $6 \%$ dos homens apresentaram esta condição. Uma possível explicação para essa constatação refere-se à distribuição de papéis na sociedade. Entre as moças, parece existir menor reforço social para a prática de exercícios físicos e maior dependência para atividades vinculadas às artes e tarefas domésticas (Taylor et al., 1999).

No que se refere à associação entre IC e NAF, a atual investigação demonstrou que, independente do sexo, a IC não está relacionada ao fato de o indivíduo ser ativo ou inativo. Adami e Vasconcelos (2008) encontraram resultados semelhantes em adolescentes de escolas públicas de Florianópolis - SC. Desta forma, os autores apontam que o NAF não influencia a insatisfação com a IC, indicando que outros fatores, dentre eles, a internalização do ideal de beleza corporal, influências do meio social e familiar e o IMC apresentam maior influência sobre a IC do que o NAF.

Com relação à associação entre a IC e o estado nutricional verificou-se que os universitários com excesso de peso foram os que apresentaram as maiores prevalências de insatisfação com a IC. Estes achados corroboram estudos realizados com universitários (Kakeshita \& Almeida, 2006), os quais aplicaram o mesmo instrumento para a avaliação da IC. Resultados semelhantes têm sido observados já no ensino médio (Petroski \& Glaner, 2009) e, de acordo com o observado na presente investigação, a tendência parece manter-se no ensino superior.

O resultado do presente estudo pode ser justificado devido a uma forte tendência cultural em considerar a magreza como uma situação ideal de aceitação social, sobretudo, no sexo feminino (Schwartz \& Brownell, 2004). Desta forma, o excesso de peso pode conduzir a um afastamento do ideal de beleza, o que reflete percepção negativa da própria IC (Stipp \& Marques de Oliveira, 2003).

Como principais limitações do estudo destacam-se: 1) o delineamento transversal, o que impede o estabelecimento de inferência causal; 2) o uso do BSQ-34 para verificar a insatisfação com a IC, pois este questionário só permite avaliar a insatisfação pelo excesso de peso.

Os resultados observados no presente estudo permitem concluir que a insatisfação com a IC está associada ao estado nutricional, sendo que os universitários com excesso de peso foram os mais insatisfeitos, quando comparados àqueles com baixo peso e peso normal. Diante disso, verifica-se que a propagação de estereótipos magros, sobretudo, para o sexo feminino, tem influenciado cada vez mais a busca por estes padrões, principalmente, entre os indivíduos com excesso de peso. Desta forma, este fato deve ser utilizado como indicação para a promoção de mudanças no estilo de vida que visem a adequação a um estado nutricional mais saudável, provocando, 
também, mudanças positivas na IC.

Ademais, sugere-se a realização de estudos experimentais que analisem a influência da prática da atividade física na insatisfação com a IC em estudantes universitários, uma vez que este assunto deve ser mais explorado para o avanço do conhecimento relacionado a esta temática na área da saúde.

\section{Agradecimentos:}

Nada declarado.

\section{Conflito de Interesses:}

Nada declarado.

\section{Financiamento:}

Nada declarado.

\section{REFERÊNCIAS}

Adami, F., \& Vasconcelos, F. A. G. (2008). Obesidade e maturação sexual precoce em escolares de Florianópolis - SC. Revista Brasileira de Epidemiologia, $11(4), 549-560$.

Alves, E., Vasconcelos, F. A. G. , Calvo, M. C. M., \& Neves, J. (2008). Prevalência de sintomas de anorexia nervosa e insatisfação com a imagem corporal em adolescentes do sexo feminino do Município de Florianópolis, Santa Catarina, Brasil. Cadernos de Saúde Pública, 24(3), 503-512.

Andrade, A., \& Bosi, M. L. M. (2003). Mídia e subjetividade: impacto no comportamento alimentar feminino. Revista de Nutrição, 16(1), 117-125.

Bosi, M. L. M., Uchimura, K. Y., \& Luiz, R. R. (2008). Eating behavior and body image among psychology students. Jornal Brasileiro de Psiquiatria, 58(3), 150-155.

Coqueiro, R. S., Borges, L. J., Araújo, V. C., Pelegrini, A., \& Rodrigues, A. B. (2009). Medidas auto-referidas são válidas para avaliação do estado nutricional na população brasileira? Revista Brasileira de Cineantropometria e Desempenho Humano, 11 (1), 113-119.

Damasceno, V. O., Lima, J. R. P., Vianna, J. M., Vianna, V. R. A., \& Novaes, J. S. (2005). Tipo físico ideal e satisfação com a imagem corporal de praticantes de caminhada. Revista Brasileira de Medicina do Esporte, 11 (3), 181-186.

Davis, C., \& Cowles, M. (1991). Body image and exercise: A study of relationships and comparisons between physically active men and women. Sex Role, 25(1-2), 33-44.

Di Pietro, M., \& Silveira, D. X. (2009). Internal validity, dimensionality and performance of the Body Shape Questionnaire in a group of Brazilian college students. Revista Brasileira de Psiquiatria, 31(1), 21-24.

Ferreira, S. E., Tufik, S., \& Mello, M. T. (2001). Neuroadaptação: uma proposta alternativa de atividade física para usuários de drogas em recuperação. Revista Brasileira de Ciência e Movimento, 9(1), 31-39.

Fontes, A. C. D., \& Vianna, R. P. T. (2009). Prevalência e fatores associados ao baixo nível de atividade física entre estudantes universitários de uma universidade pública da região Nordeste. Revista Brasileira de Epidemiologia, 12 (1), 20-29.

França, C., \& Colares, V. (2008). Estudo comparativo de condutas de saúde entre universitários no início e no final do curso. Revista de Saúde Pública, 42(3), 420-427.

Frutuoso, M. F. P., Bismarck-Nasr, E. M., \& Gambardella, A. M. D. (2003). Redução do dispêndio energético e excesso de peso corporal em adolescentes. Revista de Nutrição, 16(3), 257-263. IPAQ research commitee (2005). Guidelines for data processing and analysis of the International Physical Activity Questionnaire (IPAQ). Stockholm: Karolinska Institute.

Kakeshita, I. S., \& Almeida, S. S. (2006). Relação entre índice de massa corporal e a percepção da auto-imagem em universitários. Revista de Saúde Pública, 40(3), 497-504.

Matsudo, S., Araujo, T., Matsudo, V., Andrade, D., Andrade, E., Oliveira, L. C., \& Braggion, G. (2001). Questionário Internacional de Atividade Física (IPAQ): Estudo de validade e reprodutibilidade no Brasil. Revista Brasileira de Atividade Física e Saúde, 6(2), 05-18. 
Petroski, E. L., \& Glaner, M. F. (2009). Insatisfação corporal em adolescentes rurais e urbanos. Motricidade, 5(4), 13 - 25.

Quadros, T. M. B., Pinheiro Gordia, A., Rebolho Martins, C., Santos Silva, D. A., Pinheiro Ferrari, E., \& Petroski, E. L. (2010). Imagem corporal em universitários: associação com estado nutricional e sexo. Motriz, 16(1), 78-85.

Ribeiro, R. Q. C., Lotufo, P. A., Lamounier, J. A., Oliveira, R. G., Soares, J. F., \& Botter, D. A. (2006). Fatores adicionais de risco cardiovascular associados ao excesso de peso em crianças e adolescentes: o estudo do coração de Belo Horizonte. Arquivos Brasileiros de Cardiologia, 86(6), 408-418.

Rodrigues, E. S. R., Cheik, N. C., \& Mayer, A. F. (2008). Nível de atividade física e tabagismo em universitários. Revista de Saúde Pública, 42 (4), 672-678.

Schwartz, M. B., \& Brownell, K. D. (2004). Obesity and body image. Body Image 1 (1), 43-56.

Silva, G. S. F., Bergamaschine, R., Rosa, M., Melo, C., Miranda, R., \& Bara Filho, M. (2007). Avaliação do nível de atividade física de estudantes de graduação das áreas saúde/biológica. Revista Brasileira de Medicina do Esporte, 13(1), 39-42.
Stipp, L. M., \& Marques de Oliveira, M. R. (2003). Imagem corporal e atitudes alimentares: Diferenças entre estudantes de nutrição e de psicologia. Saúde em Revista 5(9), 47-51.

Taylor, W. C., Yancey, A. K., Leslie, J., Murray, N. G., Cummings, S. S., Sharkey, S. A., \& McCarthy, W. J. (1999). Physical activity among African American and Latino middle school girls: consistent beliefs, expectations, and experiences across two sites. Women Health, 30(2), 67-82.

Vieira, C. M., Sabadin, E., \& Marques de Oliveira, M. R. (2008). Avaliação das práticas alimentares e do estado nutricional de universitárias do primeiro ano de nutrição. Revista Simbio-Logias, $1(1), 87-98$.

Vieira, F. R., Dantas, E. H. M., Lacerda, Y., \& Novaes, J. S. (2005). Imagem corporal de obesos. Fitness e Performance journal 4(1), 19-26

Williams, P., \& Cash, T. (2001). Effects of a circuit weight training program on the body images of college students. International Journal of Eating Disorders, 30(1), 75-82.

(cc)BY-NC Todo o conteúdo da revista Motricidade está licenciado sob a Creative Commons, exceto quando especificado em contrário e nos conteúdos retirados de outras fontes bibliográficas. 